\title{
"I wish I understood this so I could make it change": Results from a Web-Based Survey Investigating Weight Loss and Regain
}

\begin{abstract}
Extant literature reports weight regain is common following many weight loss interventions. Given the poor health outcomes associated with such regain it was of interest to examine the personal trajectory of weight gain, loss and subsequent regain to unpack the complexity of obesity as a process and identify key elements of the lived experience associated with each stage. It was anticipated findings could inform future complementary and alternative therapeutic initiatives in practical ways. A 15-item web-based survey was implemented and data were analysed using a phenomenological theoretical framework. Results suggested any focus on weight loss as a central goal is problematic, unsustainable and negatively impacts mental health in specific ways, with the degree of negative affect being grossly underestimated. Further, understanding the enablers and barriers associated with weight cycling can help develop appropriate approaches to creating changes that have personal purpose and meaning, as opposed to many default perspectives that relate to food and physical activity alone. Evaluations of complementary and alternative therapeutic approaches which support healthy weight stabilisation through a sustained quality of life model are encouraged for identifying any changes in success rates, including improvements in mental health.
\end{abstract}

Keywords: Obesity; Overweight; Weight management; Weight regain; Women's health

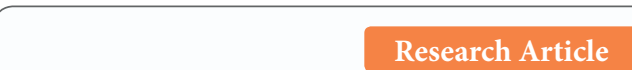

Volume 8 Issue 6 - 2017

Shane McIver* and Rasha Tayeh

Faculty of Health, Deakin University, Australia

*Corresponding author: Shane McIver, Faculty of Health, Deakin University, 221 Burwood Hwy, Burwood. Victoria. Email: shane.mciver@deakin.edu.au

Received: July 25, 2017 | Published: September 26, 2017

\section{Introduction}

Increasing rates of obesity throughout the world have augmented efforts to try and curb the global trend [1]. Despite these efforts, prevention and treatment strategies assessed through evidence-based reviews have not indicated particularly effective results with respect to reductions in body mass index (BMI), indicating this is a difficult area to produce change [2,3]. Dietary-led methods focusing on weight loss as a goal in itself, such as those involving very low calorie (kilojoule) diet programs, appear to only work in the short term $[4,5]$, questioning the longterm efficacy of these approaches.

Adhering to physical activity regimes can also be problematic, since $50 \%$ drop-out rates for individuals within six months of commencing a new program are not unusual [6]. Furthermore, one review found diet-only and diet-plus-exercise programs are often associated with partial weight regain [7]. Some pharmacological interventions have produced clinically significant levels of weight loss within six months, however follow-up reports suggest maintenance is difficult, given subsequent weight regain as well [8], especially when medication ceases [9]. Although there is evidence to suggest psychological models are capable of producing an initial phase of moderate weight loss, particularly when combined with dietary programs and physical activity, [10] a paucity of long term studies prevents any conclusions regarding results over time [11].
Although weight reduction benefits health, it is the maintenance of any reduction that proves most difficult. Shortterm interventions might achieve relative success, yet fail to sustain the results, given studies typically report returns to original weight within one to five years [12]. Such repeated patterns of weight loss and gain can be damaging. For example, for women, weight cycling has been found to be a strong predictor of greater weight gain, less physical activity and a higher prevalence of binge eating [13].

These various perspectives underline the complexity associated with obesity and the various lenses through which it is studied. Predictors for successful weight loss and maintenance have been identified to varying degrees [14-16], such as control of over-eating, motivation, social support, self-efficacy, increased levels of exercise and dietary restraint. Many individuals do not share the characteristics or tendencies required, however. Accordingly, there is a need to investigate the experience of those struggling to improve health in the absence of such traits, to inform future initiatives which might improve wellbeing and quality of life for an overweight or obese cohort.

When reviewing outcomes from weight loss or maintenance programs it is often unclear if the programs were developed with consultation from intended participants, or through imposing a well-intentioned model. The latter scenario could possibly account for attrition or disappointing findings during any post- 
treatment follow-up. As such, there is a strong rationale for examining the lived experience of those who have attempted and achieved weight loss, but also and more importantly, to consider the experiential mechanisms associated with the subsequent regain, given this is often an inevitable outcome of many programs with a weight-loss focus.

The range of qualitative and quantitative studies which have examined weight regain tend to consider the social, physical or psychological correlates of weight regain separately, to the exclusion of the other $[17,18]$. As some research has noted, the emergence of a biopsychosocial, body-mind understanding of weight regain is timely $[19,20]$, yet remains to be fully explored. Therefore, the present study sought to examine the lived experience of obesity and weight regain, to obtain greater understanding about the difficulties associated with weight maintenance following weight loss. It was anticipated findings could inform future interventions or health promotion programs in ways which have possibly not yet been fully identified or developed.

\section{Methods}

Qualitative data was sought from those who were overweight or obese, lost weight, and who subsequently regained. In conjunction with computer programmers, the researchers drew on web-based methods for disseminating a 15-item survey [21]. Data collection from online surveys is a useful way to obtain sensitive information, because respondents can remain anonymous. Further, an empirical analysis of online research concluded that 'the data provided by internet methods are of at least as good quality as those provided through traditional paper and pencil measures'[22].

Prior to release, the survey was piloted among a small group to establish content and face validity. Examples of questions were "What prompted you to start to lose weight, or consider losing weight? What was the first step in the process- how did it come about?" and "How did you go about losing gained weight?" as well as an open format section on thoughts and reflections. Questions encouraged participants to report on the steps of initial weight loss followed by items relating to subsequent weight gain.

Recruitment efforts included issuing press releases to primary and secondary electronic and print media, referring participants to a website containing information about the survey, including confidentiality and consent processes as per international human ethics requirements. Specifically, the home site of the survey explained the study with a check box to provide informed consent and to progress to completing the survey. Participants had the option for providing contact information at the end of the survey if they wanted to provide feedback from findings as a form of respondent validation. In alignment with comparable research [23], to qualify for the study individuals needed to be over 21 years of age and have initially lost at least $5 \%$ and subsequently regained at least $5 \%$ of their baseline weight.

\section{Participants}

Data was collected from a non-clinical community sample of 30 participants (29 women, 1 man) from urban $(n=21)$ and regional $(n=9)$ Australia who were the first to engage with the 15-item survey online. Of the initial 30 respondents in the survey, the estimated heaviest weight ranged between 47 to $168 \mathrm{kgs}(\mathrm{M}=$ $98.42, \mathrm{SD}=25.5)$, with the estimated lowest weight ranging from 33 to $151 \mathrm{kgs}(\mathrm{M}=71.21, \mathrm{SD}=22.5)$. The mean for the cohort's current weight (range $38-130 \mathrm{kgs}$ ) was $88.26 \mathrm{kgs}$ (SD = 23.2). Ages ranged from $22-66$ years, with a mean age of 45 years $(\mathrm{SD}=$ 11.6).

\section{Data analysis}

Analysis was informed by a phenomenological theoretical framework, given the researchers were interested in the lived experience of obesity, weight loss and regain. Bracketing and use of the hermeneutic spiral were enlisted to augment rigor [24]. Accordingly, personal biases were identified prior to and throughout the analysis procedure to minimize any potential influence. Data were considered within and across responses from the overall cohort as well as within and across individual responses and compared accordingly to identify theme clusters and ultimately, key themes [25]. For most questions, data saturation occurred after approximately 20 responses, sometimes even sooner. Findings were reported back to five participants who had provided contact details as a form of respondent validation [26]. During this latter process, results were affirmed and no conflict with the findings was identified.

\section{Results}

Given the survey questions chart a linear progress over the course of weight gain, loss and subsequent regain, as well as overall thoughts and reflections, reported findings followed the same course of these four main categories, with dominant, overlapping and recurring themes and characteristics identified throughout.

\section{Weight gain}

a. "I ate my feelings": When eating as a solution becomes the problem. When asked what made participants gain weight initially, emotional eating was often cited. Eating was typically sought as a solution to feeling bad, whether about oneself or one's circumstances. Comfort eating was associated with eating foods which were not necessarily nurturing from a nutrition perspective, but to placate negative feelings for the duration of the eating period. As one respondent reported, ". . . as I went further into my shell, my hand went deeper into the snack box." A willingness to socialize diminished as the desire for comfort eating increased. As such, food as a coping mechanism and a solution to life's problems, even if momentary, evolved into becoming one of the central problems over time.

i. The fog of no direction, disconnection and depression: Participants also self-identified low self-esteem, self-loathing, and depression as contributing to weight gain, often referring to the chronic nature of these perceptions, typified by comments such as "never feeling good about myself." Feelings of being directionless, bored, having a disrupted routine or a relentless one, all promoted overeating as a distraction, enabling a deeper spiral into self-neglect. Participants witnessed emotional turmoil and negative repercussions with awareness, yet felt somewhat powerless at the time to steer an alternate course in 
decision-making. During such times, illogical decisions such as the conscious preference for "bad food" prevailed.

ii. Stress and fatigue: Although stress and fatigue were reported as contributing to weight gain, it was as if a freefloating anxiety provided a scaffold for the stress and fatigue to manifest and flourish. Anxiety, stress and fatigue were all identified as chronic (rather than acute) states. The stressors were not necessarily task oriented, but rather attributed to engaging with thought processes linked to negativity, specifically negative self-talk. Underlying tones of constantly berating the self and critical self-judgment permeated most responses. A constant stream of negative internal dialogue was fueling feelings of exhaustion, countering any efforts to create meaningful change.

\section{Weight Loss}

a. "Something just happens and I hate myself": The tipping point. Participants identified a tipping point where a pain threshold of dissatisfaction and despair was reached, catalyzing the desire for change. This usually happened through two principal streams, both involving negative experiences: a) either a reaction to an internal, experiential event, or b) a reaction to an external event. Examples of internal experiences included clothes not fitting, joints causing problems, specific feelings such as shame and embarrassment or generally feeling bad. Examples of external events included seeing one's reflection, one's appearance in a candid photograph, or looks and comments from other people, the latter frequently reported as being the most hurtful. The desire for health in and of itself was not usually mentioned as a catalyst for considering weight loss. Various forms of self-disgust, however, which magnified discomfort in ways which were no longer tenable, often triggered seeking pathways for radical and immediate change.

Even though respondents mentioned addressing "diet and exercise", these generic terms were rarely deconstructed or explained. It was as if referring to such general terms sufficiently expressed what weight-loss involves. There was no mention of expectations relevant to each, just that pursing complete changes in diet (somehow) and exercise (somehow) were inevitable requirements.

Participants rarely questioned their approach to weight loss, or the specific information they adopted as their guide. Most joined gyms. Fewer joined weight-loss organizations, with two respondents choosing weight-loss products or programs available from outlets such as chemists. A striking feature of this process is how participants seemingly devised strategies of their own making, based on what they considered to be correct approaches, informed or otherwise. There was little mention of liaising with dieticians or health program specialists on an ongoing basis. One participant mentioned working with a personal trainer, only to abandon the initiative later.

Participants swung from the experience of being out of control to enforcing complete self-control, encouraged by the early results weight loss delivered. These included "feeling younger," "liberated," and "empowered." These observations were typically accompanied by other comments which indicated awareness that at such positive outcomes were unsustainable, such as ". . . felt fantastic, but couldn't keep up the diet forever," and from another: "It was sheer hell . . . very bad tempered . . . but has always been hair-triggered." One participant noted: "I found the whole experience frustrating, boring but rewarding when I achieved my goals." Participants liked the idea of what weight loss seemingly promised; increased energy and confidence, positive feedback from others, being noticed and the concept that weight loss would inevitably equate to being a better person. Indeed, many of these positive outcomes were experienced in the early stages. However, the euphoria was often short-lived. Ultimately, comments described being disenchanted with what the process of what weight loss seemed to entail in the long term. For example, ". . . felt no different. Did not feel stronger. Emotionally lived on the edge always worried about gaining weight, even a few grams."

The most satisfying form of weight loss appeared to arise when losing the weight was not a focus, but rather a by-product from changing one's life. For example, one respondent moved to another country, and in the absence of reminders and triggers of old habits, experienced circumstantial weight loss: "When I was at my 'ideal' weight, I guess I wasn't focused on my weight, and therefore didn't waste time thinking about it and could focus on more important issues." For this participant, weight regain occurred after returning to the same familiar places and reestablishing familiar habits and associations.

b. Crunching the numbers: When responding to questions regarding the amount of weight lost over time from these efforts, participants were extremely clear and specific, being able to cite exact amounts over what period of time and in what year, such as this observation offered by one respondent, “... lost $27 \mathrm{k}$ over two years then a further 10 kilos after a break of three years over a five month period," and by another: ". . . 12 months took off $11 \mathrm{kgs}$ back in 1995." Most could even state circumstances or age range relevant to the corresponding weight loss. It was as if the loss represented a singular achievement frozen in time. As one participant reported, " . . . never felt the amount I had lost was good enough. Never felt thin." Although previous weight loss occurred slowly (in a healthy time frame), the fact that weight loss should be fast and dramatic remained an expectation.

\section{Weight regain}

a. "I went through a period where I simply lost the plot": Tyrant versus rebel. Participants typically developed various models of weight loss that proved difficult to sustain. On the surface, participant weight gain could be summarized as eating more and moving less over time. At a deeper level, participants acknowledged embarking on a weight loss project was an insurmountable internal battle. Phrases such as "emotional rollercoaster" and "wrestling with myself" provided insights to the serious and internal conflicts confronted throughout the process. As one participant noted, "it's very hard work and can add to depression."

Given observations in the data, it appears at this stage negative self-talk manifested in two distinct forms, being both "tyrant" and "rebel". The "tyrant" develops a regime where diet and exercise 
are apparently used as punishment for "being fat". In response to the nagging dissatisfaction of how one looks and feels, the "tyrant" aspect devised punishing diets and disproportionately demanding workouts with programs so ambitious they were exhausting to complete. In time, as the workout of one's own creation became increasingly overwhelming, the response was to rebel and do nothing. The same approach applied to food. The "tyrant" enforced strict regimens which the "rebel" abhors and ultimately rejects. As one participant noted:

b. I was getting sneaky with food: I would buy more than was being prepared for me to compensate for what I thought I needed. I was back eating what I was when I was at my heaviest and unhealthiest. The more I ate, the harder I found exercise to do.

These ongoing conflicting messages created a lack of energy, building to intense exhaustion. Participants mentioned the fatiguing process of constant vigilance, "keeping tabs on myself," and when the vigilance slipped, feelings of being out of control surfaced once again. The desire to escape the negativity was also evident, with statements such as, "I wish I understood this so I could make it change."

c. Slow loss, speedy gain: For most participants, subsequent weight regain was unnervingly rapid. In addition, while participants were acutely aware weight was increasing, they experienced a form of inertia which prevented them from adopting any action. One participant wrote, "Miserable. I knew it was happening but couldn't bear to go back on the diet for some reason." The question of whether weight loss was worth the effort arose often, especially given what participants went through to obtain even minor losses. As one participant observed, "I find it hard to think about anything other than how fat I am."

d. The broken spirit: Patterns of seclusion and withdrawal typically resurfaced for participants during weight regain, accompanied with feelings of being a failure. Feelings of self-loathing intensified with each unsuccessful attempt. Comments included, "I feel I have let myself down," "felt cheated and annoyed," "I want to stay home and hide," "angry at myself for being so big" and "I hate myself." One participant wrote, "if [I'm] unattractive, people will expect less of me so I can eat and sleep more."

Responses to weight gain dramatically reflected a despairing state; a place of being "emotionally hurt," "unattractive," "sad," "lonely," "lethargic" and "depressed." The demoralizing nature and frustration of weight regain was considered unbearable, along with obligatory attempts at future change that inevitably devolved into a series of false-starts and dismay at the multiple resolves to diet and exercise which never eventuated. One participant wrote, "if I could channel this wasted energy into the issues in my life that truly need attention, then I'd be a lot better off."

\section{When nothing works}

a. "Putting on the weight seems like my whole life": Addiction to the process. Comparable to the vulnerability experienced by the participants during weight regain was the frustration of the plateau. This was a state of limbo and feeling stuck, where nothing seemed to shift, regardless of any action, be it dietary change or exercise. In this state, possible solutions proved elusive and how to progress was not identified or known. There was simply the feeling of having to endure stagnation for however long it lasts. Participants experiencing this stage were exasperated and wrote about seriously considering interventions such as lapband surgery.

b. Repeating the patterns: When questioned at the end of the survey about current weight-related goals, objectives or observations, most participants reverted to citing the same goals and aspirations they had previously mentioned, including the methods they would adopt to attempt achieving them, despite the evidence their past attempts and approaches were not successful. Comments included awareness of the emotional toll the process takes, such as the need to be ever vigilant. Participants noted the requirement for lifestyle changes and the transformation of old habits, such as "moderation" and "being kinder to myself" while acknowledging difficulties associated with how those changes might be established: "Sometimes the strength I need to diet is just overwhelming."

\section{Discussion}

Findings extend current research through identifying the degree to which patterns of weight loss and regain negatively impact mental health. Contrary to social and typical expectations that weigh loss is celebrated, data indicated that once weight loss becomes a central focus, a psychological nightmare begins. Most participants experienced the process of engaging with weight loss goals as a form of overwhelming and debilitating quicksand. All attempts to struggle against its disabling forces to keep afloat simply contributed to the fatigue of trying to maintain the effort.

Contrary to what participants believed, the sole pursuit of weight loss appears to not be as powerful or as helpful as pursuing life interests which have purpose and meaning to each individual. Indeed, data suggested weight loss might be a by-product of this latter process, addressing the original intent, but in a personally fulfilling way. Freed from the reduction of weight as an end-point, other freedoms arose.

Without the weight loss polarities of success and failure as a central, internalized dilemma, negative self-talk can diminish. The language of self-loathing from participants was startlingly pervasive and repetitive within and across the dataset. Although negative self-affect is mentioned in weight loss literature [14], the proliferation and intensity of various references to self-hate in the present study was not anticipated. As a process, it is as if focusing on weight loss did not allow individuals to relate to oneself other than deficient. For the study's participants, exercise and dieting models were repressive and difficult to adhere to. Unrealistic challenges resulted in all effort being negated by feeling overwhelmed. Self-designed programs and expectations did not typically match evidence-based best-practice approaches or reasonable outcomes [8], given rapid weight loss was consistently anticipated. A pattern of rebelling against self-imposed restrictive 
regimes was identified in the data, ultimately contributing to negative self-affect, feelings of failure, hopelessness and despair. Improved health was not considered a principal motivating factor to lose weight, conflicting with a large proportion of research arguing the case for health status and the development of riskfactors as a central tenet for weight reduction. Rather, the desire to diminish the pain of negative emotions and to feel and look better, especially in relation to functioning, wearing certain clothes, and generally relating to one's self in better ways, were more influential factors, emphasising how improving quality of life (in lieu of weight loss) is an important primary aim.

Solutions to promoting weight stabilisation remain elusive. Gaps in efficacy of traditional approaches pave the way for complementary and alternative initiatives to be developed, implemented and evaluated. For example, there is promising evidence demonstrating the capacity for meditation-based approaches to concurrently treat co-morbidities associated with disordered eating and poor mental health outcomes such as the anxiety reported in the present study [27-29].

As noted, participants found the success of any weight loss was immediately countered by the dread of regain, accompanied with ongoing and negating self-talk and derision. It is possible programs that help individuals objectively witness these negative patterns of cause and effect could act as circuit-breakers to cease selfsabotaging thoughts and behaviours, as per the multiple outcomes reported in mindfulness meditation literature [30,31]. Similarly, modalities that encourage healing fractured relationships to the body through enjoying the body more and worrying about it less are required to counter the reported tendencies where participants punished the body through strict and unquestioned regimes. Once again, complementary and alternative therapeutic approaches appear to support this transition and possibly have the capability to be tailored accordingly [32-34]. Given relevant reviews often state further research is required to substantiate positive early evidence, this should inspire augmented efforts to rigorously evaluate new and alternative initiatives that deviate from mainstream models of weight maintenance. Accordingly, it is anticipated findings from the present study will be useful to inform appropriate program development and outcome measures.

In terms of limitations, participants self-selected to engage with the survey, and this can be interpreted as skewing the data. Small datasets can also be viewed as non-representational. These quandaries occur in most qualitative research, so these debates are common. Ethics requirements promote self-selection in the absence of randomisation, however, and the resultant data offered considerably thick and rich description [26]. Also, data saturation suggested many experiences were shared, and therefore could well apply to a broader sample. It was not possible to identify any potential gender differences, given respondents were mostly women. However, since weight cycling for women is particularly problematic, such representation was useful [35].

\section{Conclusion}

Results suggest individuals do not necessarily reflect on the sabotaging effects of multiple weight loss attempts. Participants often negated their own observations and experiences in preference for repeatedly deferring to persuasive discourses which reinforce weight loss and the thin ideal as central goals. Despite this recurring pattern, these dominant discourses were rarely challenged. In such circumstances, feelings of failure became normative. In comparison, participants experiencing positive outcomes found weight loss to be a by-product of pursuing life interests that had personal purpose and meaning.

For healthcare professionals aiming to improve the quality of life for those who are overweight or obese, current challenges include how to translate empowering principles and practices into appropriate action through more holistic approaches. Future research comparing the efficacy of these approaches to traditional weight loss models is encouraged.

\section{Funding}

The authors gratefully acknowledge funding support from VicHealth, who had no role in the study design, collection, analysis or interpretation of the data, or writing the manuscript.

\section{Conflict of Interest}

This submission has no conflicting interests. It is the original work of the authors and has not been submitted for publication elsewhere.

\section{Contributors}

The corresponding author designed the study and wrote the initial manuscript draft. Author B conducted literature searches and provided summaries of previous research studies. Both conducted the thematic analysis and both contributed to and have approved the final manuscript.

\section{References}

1. World Health Organization (2011) Obesity and overweight: Fact sheet $N^{\circ} 311$. World Health Organization, Geneva, Switzerland.

2. Loveman E, Frampton GK, Shepherd J, Picot J, Cooper K, et al. (2011) The clinical effectiveness and cost-effectiveness of long-term weight management schemes for adults: a systematic review. Health Technol Assess 15(2): 1-182.

3. Ostman J, Britton M, Jonsson E (2004) Treating and preventing obesity: An evidence based review. Wiley-VCH, Germany.

4. Dansinger ML, Tatsioni A, Wong JB, Chung M, Balk EM (2007) Metaanalysis: the effect of dietary counseling for weight loss. Ann Intern Med 147(1): 41-50.

5. Tsai AG, Wadden TA (2006) The Evolution of Very-Low-Calorie Diets: An Update and Meta-Analysis. Obesity (Silver Spring) 14(8): 12831293.

6. McElroy M (2002) Resistance to Exercise: A social analysis of inactivity. Human Kinetic, Champaign, USA.

7. Wu T, Gao X, Chen M, van Dam RM (2009) Long-term effectiveness of diet-plus-exercise interventions vs. diet-only interventions for weight loss: a meta-analysis. Obes Rev 10(3): 313-323.

8. Ulen CG, Huizinga MM, Beech B, Elasy TA (2008) Weight Regain Prevention. Clinical Diabetes 26(3): 100-113.

9. Bray GA (2005) Drug treatment of obesity. Psychiatry Clinics of North America 28: 193- 217. 
10. Thompson WG, Cook DA, Clark MM, Bardia A, Levine JA (2007) Treatment of Obesity. Mayo Clinic Proceedings 82(1): 93-102.

11. Shaw K, O’Rourke P, Del Mar C, Kenardy J (2006) Psychological interventions for overweight or obesity. Cochrane Database Syst Rev 18(2): CD003818.

12. Biedert E (2005) The psychological and pharmacological treatment of binge eating disorder: An overview. (Ed.), Obesity and Binge Eating Disorder. Karger 171: 165-179.

13. Field AE, Manson JE, Taylor CB, Willett WC, Colditz GA (2004) Association of weight change, weight control practices, and weight cycling among women in the Nurses' Health Study II. Int J Obes Relat Metab Disord 28(9): 1134-1142.

14. Del Corral P, Bryan DR, Garvey WT, Gower BA, Hunter GR (2011) Dietary adherence during weight loss predicts weight regain. Obesity 19(6): 1177-1181.

15. Elfhag K, Rössner S (2005) Who succeeds in maintaining weight loss? A conceptual review of factors associated with weight loss maintenance and weight gain. Obesity Reviews 6(1): 67-85.

16. Wing RR, Papandonatos G, Fava JL, Gorin AA, Phelan S, et al (2008) Maintaining large weight losses: the role of behavioral and psychological factors. J Consult Clin Psychol 76(6): 1015-1021.

17. Byrne S, Cooper Z, Fairburn C (2003) Weight maintenance and relapse in obesity: a qualitative study. Int J Obes Relat Metab Disord 27(8): 955-962.

18. MacLean PS, Higgins JA, Giles ED, Sherk VD, Jackman MR (2015) The role for adipose tissue in weight regain after weight loss. Obes Rev 16(Suppl 1): 45-54.

19. Ogden J, Avenell S, Ellis G (2011) Negotiating control: Patients' experiences of unsuccessful weight-loss surgery. Psychol Health 26(7): 949-964

20. Yankura DJ, Conroy MB, Hess R, Pettee KK, et al. (2008) Weight regain and health-related quality of life in postmenopausal women. Obesity 16(10): 2259-2265

21. Dillman DA, Smyth JD (2007) Design effects in the transition to web-based surveys. . American Journal of Preventive Medicine 32(5 Suppl): S90-S96.

22. Gosling SD, Vazire S, Srivastava S, John OP (2004) Should we trust web-based studies? A comparative analysis of six preconceptions about internet questionnaires. Am Psychol 59(2): 93-104.
23. Engel SG, Crosby RD, Kolotkin RL, Hartley GG, Williams GR, et al. (2003) Impact of weight loss and regain on quality of life: Mirror image or differential effect? Obes Res 11(10): 1207-1213.

24. Fischer CT (2009) Bracketing in qualitative research: Conceptual and practical matters. Psychother Res 19(4): 583-590.

25. MilesMB, Huberman AM (1994) Qualitative Data Analysis: An Expanded Sourcebook. (2 ${ }^{\text {nd }}$ edn), Sage Publications, Newbury Park, USA.

26. Patton MQ (2002) Qualitative Research and Evaluation Methods. Sage Publications, Thousand Oaks, USA.

27. Goyal M, Singh S, Sibinga EM, Gould NF, Rowland-Seymour A, et al (2014) Meditation programs for psychological stress and well-being: A systematic review and meta-analysis. JAMA Internal Medicine 174(3): 357-368.

28. Katterman SN, Kleinman BM, Hood MM, Nackers LM, Corsica JA (2014) Mindfulness meditation as an intervention for binge eating, emotional eating, and weight loss: a systematic review. Eat Behav 15(2): 197-204.

29. O’Reilly GA, Cook L, Spruijt-Metz D, Black DS (2014) Mindfulnessbased interventions for obesity-related eating behaviours: a literature review. Obes Rev 15(6): 453-461.

30. Hoge EA, Bui E, Marques L, Metcalf CA, Morris LK, et al. (2013) Randomized controlled trial of mindfulness meditation for generalized anxiety disorder: effects on anxiety and stress reactivity. J Clin Psychiatry 74(8): 786-792.

31. Tang YY, Hölzel BK, Posner MI (2015) The neuroscience of mindfulness meditation. Nat Rev Neurosci 16(4): 213-225.

32. Büssing A, Michalsen A, Khalsa SB, Telles S, Sherman KJ (2012) Effects of Yoga on Mental and Physical Health: A Short Summary of Reviews. Evid Based Complement Alternat Med 2012: 165410.

33. Kahlsa SBS 2007) Yoga as a Therapeutic Intervention. In: PM Lehrer et al. (Eds.), Principles and Practice of Stress Management ( $3^{\text {rd }}$ edn), The Guildford Press, New York, USA, pp. 449-462.

34. Neumark-Sztainer D (2014) Yoga and eating disorders: is there a place for yoga in the prevention and treatment of eating disorders and disordered eating behaviours? Advances in Eating Disorders 2(2): 136-145

35. Byrne SM, Cooper Z, Fairburn CG (2004) Psychological predictors of weight regain in obesity. Behav Res Ther 42(11): 1341-1356. 